\title{
A Self-Consistent Model for Negative Glow Discharge Lasers: The Hollow Cathode Helium Mercury Laser
}

\author{
Gregory J. Fetzer, Member, IEEE, and Jorge J. Rocca, Member, IEEE
}

\begin{abstract}
A model for negative glow metal-vapor ion lasers that self-consistently describes the dynamics of the negative glow and the cathode sheath regions of the discharge has been developed. The model computes the electron energy distribution and the population of relevant excited states in the negative glow self-consistently with the charged particle fluxes and electric field distribution in the cathode sheath. Its application to the study of the helium-mercury charge transfer ion laser is reported. The model accurately depicts the operation of a hollow cathode in the laboratory, where for a defined cathode geometry and material, the discharge characteristics are determined by the selected discharge voltage and the gas pressure. The laser output power calculated as a function of the discharge parameters is in good agreement with experimental measurements reported in the literature. The model can be modified to simulate other negative glow discharge lasers, such as electron-beam pumped $\mathrm{CW}$ ion lasers.
\end{abstract}

\section{INTRODUCTION}

$\mathrm{T}$ HE negative glow region of a gas discharge, in which ionization and excitation is dominantly produced by energetic beam electrons, has been used successfully to excite a large number of continuous wave $(\mathrm{CW})$ metal vapor ion lasers [1]-[18]. The spatial extent of the negative glow region of a gas discharge is determined by the reaching distance of the beam electrons exiting the cathode sheath. Several types of discharge configurations have been designed to provide negative glows with a sufficiently long optical path for the generation of laser radiation. Hollow cathode discharges (HCD) have been used to generate a large variety of $\mathrm{CW}$ metal vapor ion lasers with wavelengths extending from the midinfrared to as short as $224 \mathrm{~nm}$ [1]-[16]. Extended negative glows generated by multikilovolt glow discharge electron beams have been used to generate $1.2 \mathrm{~W}$ of $\mathrm{CW}$ laser radiation in the visible from $\mathrm{Zn}$ II transitions [17] and more recently to obtain $\mathrm{CW}$ laser action from metal vapor in the ultraviolet region of the spectrum [19]. Recombination lasing has also been demonstrated in negative glow plasmas [18].

Manuscript received May 29, 1991; revised December 12, 1991. This work was supported in part by a N.S.F. Presidential Young Investigators Award to J. J. Rocca, Grant ECS-8606226 and by a N.S.F. Grant ECS9013372.

G. J. Fetzer is with the Lear Siegler Measurement Controls Corporation, 74 Inverness Drive East, Englewood, CO 80112.

J. J. Rocca is with the Department of Electrical Engineering, Colorado State University, Ft. Collins, CO 80523.

IEEE Log Number 9201376.
In negative glow discharges the characteristics of the glow plasma and of the cathode sheath regions are closely interrelated. Consequently, a complete model of these lasers requires a self-consistent treatment of these two discharge regions. Despite extensive experimental work in negative glow discharge lasers only individual aspects of negative glow discharges and negative glow laser devices have been studied theoretically [15], [20], [21] and until now a complete quantitative analysis of the devices has not been reported.

In this paper we discuss a self-consistent model of a hollow cathode discharge helium mercury laser. Running the model resembles operating a hollow cathode device in the laboratory. The variables which can be controlled by the experimentalist; the cathode geometry and material, the discharge voltage, and the partial pressures of the buffer gas and the metal vapor, are the same parameters that must be specified to run the model. Using these inputs the model self consistently calculates the discharge current, the electron energy distribution, the ion density and the population of relevant excited states. The population of the $7 p^{2} P_{3 / 2}$ and the $7 s^{2} S_{1 / 2}$ excited states in $\mathrm{Hg}$ II are calculated and subsequently used to determine the optical gain of the discharge at $6150 \AA$ and the laser power for a specified resonator geometry.

While the results presented here correspond to simulations of a helium-mercury hollow cathode laser, the selfconsistent treatment of the cathode sheath and negative glow that we describe can also be modified to model other types of negative glow lasers, such as the electron-beam excited $\mathrm{CW}$ ion lasers.

\section{A. The Hollow Cathode Discharge}

The HCD is primarily composed of two distinct regions; the cathode sheath and the negative glow, which feed each other to sustain the discharge. At pressures typical for HCD laser operation the cathode sheath size is small in comparison to the negative glow region. However, the majority of the discharge voltage is dropped across the cathode sheath and the negative glow region is practically electric field free. The strong electrical field in the sheath region accelerates ions toward the cathode and electrons toward the negative glow region. Ions produced by electron-neutral collisions in the glow diffuse into the 
sheath and are accelerated towards the cathode. Ion bombardment of the cathode results in the emission of electrons [22], [23]. These secondary electrons are accelerated through the sheath and part of them arrive at the sheath-glow boundary with an energy corresponding to the full cathode fall voltage. As such, the electron energy distribution in a hollow cathode is strongly non-Maxwellian and has a significant beam component [24], [25]. The negative glow also contains a large density of cold electrons resulting mainly from thermalized secondary electrons created during electron impact ionization of neutral atoms.

Several researchers have made calculations regarding various aspects of hollow cathode discharges and other electron beam sustained glow discharges. For instance Kagan et al. completed a four part series of papers on the electron energy distribution in the negative glow region of a helium hollow cathode discharge [21]. In their work, equations for the electron energy distribution were developed and results were obtained using approximate analytical solutions of these equations. Warner modeled the negative glow region of a hollow cathode discharge in helium assuming the influx of electrons arriving with the full cathode fall energy [20]. Based on this assumption he computed the electron energy distribution in the negative glow region. Warner examined the roles of the various electron collision mechanisms on the structure of the electron energy distribution. Using the calculated distribution he computed the excitation and ionization rates in the discharge and made comparisons to experimental measurements. Fetzer et al. used a technique similar to Warner's to model the negative glow regions of electron-beam sustained discharges in argon for the purpose of examining the feasibility of obtaining improved efficiencies by exciting the well known Ar II blue-green laser transitions via an electron beam [26].

In reference to the cathode sheath region, Davis and Vanderslice have computed the flux spectra of ions arriving at the cathode of a negative glow discharge [27]. They modeled the flow of ions in the cathode dark space assuming a linearly decreasing electric field in the region from the cathode to the negative glow boundary to obtain ion energy distributions at the cathode. The agreement between their experimental and theoretical results was excellent. They did not however extend the computations to include electron flow through the sheath region. McClure et al. [28] developed a theoretical model of the cathode sheath in high voltage deuterium discharges. Here the continuity equations for charged particles and Poisson's equation were used to compute the electric field in the sheath. Recently, Den Hartog et al. conducted both theoretical and experimental studies of the cathode region of a helium glow discharge [29]. They used a null collision Monte Carlo technique to model electron flow through the cathode sheath to allow for the computation of ionization and excitation rates. A linear electric field profile obtained through optogalvanic spectroscopy experiments was used in their calculations. They used a combination of experi- mental and theoretical results to infer the electron density in the cathode fall region. Bayle et al. have conducted in depth theoretical studies of the cathode sheath and negative glow regions of glow discharges in which the discharge gas was $\mathrm{CO}_{2}$ [30]. They developed a spatiotemporal model of a glow discharge which was based on the solution of continuity equations for ions and electrons and Poisson's equation. A Maxwellian electron energy distribution was assumed to calculate ionization and excitation rates. Specifically, they examined the roles of the electric field and collisions in determining the electron energy and momentum distributions in the sheath and negative glow regions of the discharge.

Models have been developed to analyze individual aspects of HCD lasers. Warner provided a study of the production of metal vapor by sputtering in a hollow cathode noble gas-metal vapor laser [31]. Solanki calculated excited state densities in the negative glow region of a neoncopper HCD by assuming that the electron energy distribution in the glow was a delta function at the full discharge voltage [15]. Neither of these modeling efforts represent a self consistent treatment of the HCD laser.

In this paper the electron energy distribution in the negative glow region of a noble gas-metal vapor HCD is calculated self-consistently with the energy spectrum of charged particles in the cathode sheath. The model discussed here has been used to compute the dependence of the laser output power of a hollow cathode helium-mercury laser operating on the $6150 \AA$ transition of singly ionized mercury on the discharge parameters. The results are compared to the experimental measurements made by Piper and Webb [6]. To the authors' knowledge this is the first self-consistent model of a negative glow discharge laser.

\section{B. The Helium-Mercury Laser}

Laser emission from the $6150 \AA$ transition of singly ionized mercury in a helium-mercury mixture was first reported by Bell in 1964 in a pulsed positive column arc discharge laser system [32]. Mercury vapor was diffused through helium by cataphoresis. Bell extended his work to obtain laser oscillation in a pulsed hollow cathode discharge in 1965 [2]. CW oscillation on the $6150 \AA$ transitions was subsequently obtained in a slotted hollow cathode by Schuebel in 1971 [3]. Measurements of laser output power were not reported in that paper.

Ferrario obtained a $\mathrm{CW}$ output power of $10 \mathrm{~mW}$ from the $6150 \AA \mathrm{HgII}$ transition using a positive column discharge [33]. The laser output power of Ferrario's device exhibited a rapid turnover with increasing current density. This characteristic was claimed to be a result of depletion of the ground state $\mathrm{Hg}$ atom concentration due to increased ionization at high currents. However, the problem was not remedied by increasing the vaporization rate in the metal reservoir. This effect is reflected in the results published by Ferrario in which he varied the mercury density while keeping the other operating parameters con- 
stant. A sharp turnover in laser output power was observed with increasing mercury density. The decrease in laser power occurs because the addition of mercury vapor to a helium positive column discharge cools the average electron temperature, thereby decreasing the helium ionization rate. This is a consequence of the lower ionization energy, larger ionization cross section and smaller diffusivity of mercury compared to helium. Thus, the addition of metal vapor to a positive column discharge cools the electron energy distribution and thus limits the power of noble gas-metal vapor ion lasers.

In contrast, the electron energy distribution in a HCD is not severely affected by the introduction of metal vapor. Piper and Webb constructed a helium-mercury laser excited by a hollow cathode discharge [6]. In their device the $6150 \AA$ laser output power did not exhibit a turnover with increasing current density up to dc currents of $3.5 \mathrm{~A}$ or with pulsed current excitation up to $10 \mathrm{~A}$. Detailed modeling of the device was not reported and scaling issues were not studied. A maximum laser power of approximately $70 \mathrm{~mW}$ was obtained at the maximum discharge current of $10 \mathrm{~A}$. More recently a $\mathrm{CW}$ laser power of $250 \mathrm{~mW}$ was obtained from the same transition by Rocca et al. in a negative glow discharge excited by a high voltage electron beam [17].

\section{THE ModeL}

To allow a comparison with experimental results described in [6] the device modeled in this work was selected to be a $50 \mathrm{~cm}$ long stainless steel hollow cathode with a $3 \mathrm{~mm}$ inside bore diameter. Fig. 1 illustrates the location of the negative glow and the cathode sheath on a cross section of the laser tube. Also shown in Fig. 1 is the reference frame used in the model.

The model structure is segmented into four interconnected blocks. Fig. 2 is a flow diagram of the numerical implementation of the model. The first block describes the negative glow region of the discharge. The second is for the sheath-negative glow boundary and serves primarily as a numerical interface between the computational aspects of the negative glow and the cathode sheath. The third block describes the cathode sheath region. The optical gain, laser output power, and laser efficiency are calculated in the fourth block of the model.

\section{A. The Negative Glow Region}

The negative glow, assumed to be spatially uniform in this work, is described by a zero-dimensional model consisting of the Boltzmann equation for electrons and a set of coupled, steady-state, rate equations for the ion and excited state population densities.

The technique used to solve for the electron energy distribution, denoted herein as $N_{e}(E)$, is based on the numerical solution of the Boltzmann equation for electrons and was originally developed by Peterson, [34] and subsequently used by Warner [20] and Fetzer et al. [26]. The technique exploits the fact that the negative glow region

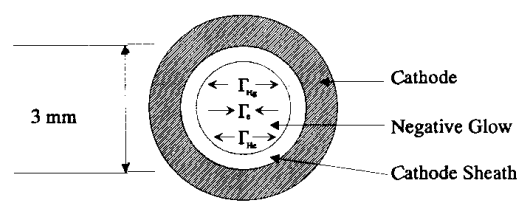

(a)

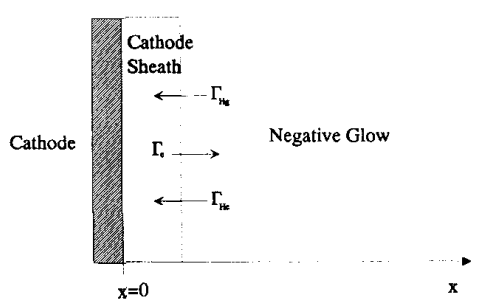

(b)

Fig. 1. (a) Geometry of negative glow and cathode sheath in the HCD device described in [6] and modeled here. (b) Reference frame utilized in one dimensional model for the cathode sheath. $\Gamma_{\mathrm{He}}, \Gamma_{\mathrm{Hg}}$, and $\Gamma_{e}$ represent the flow of ions and electrons within the cathode sheath

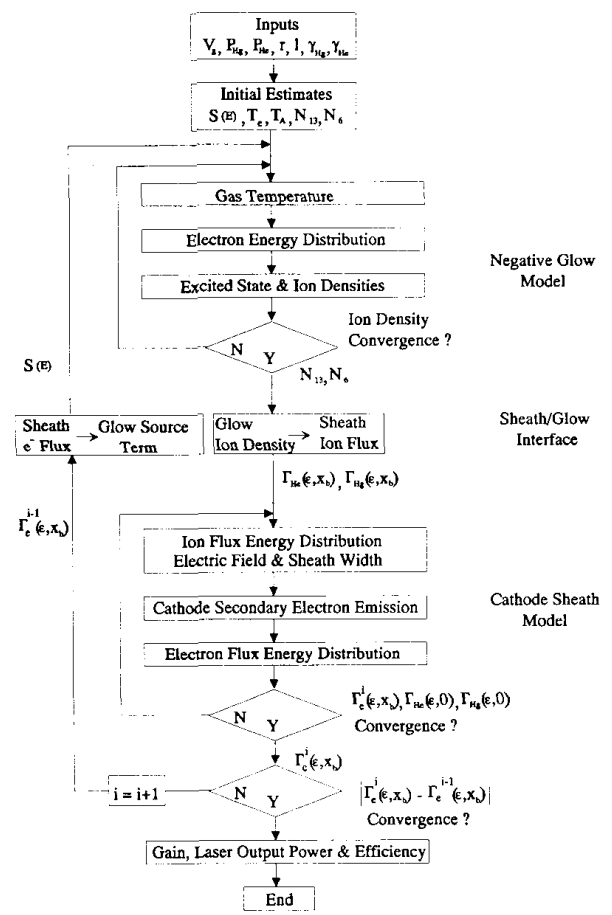

Fig. 2. Flow chart illustrating the four blocks of the model and the flow of variables among the blocks.

of the discharge is practically free of an electric field. As such, electrons introduced into the negative glow are not subject to an accelerating force but change energy through collisions with atoms, ions, and other electrons. By discretizing the energy space into a set of bins, the flow of electrons from one energy bin into others is calculated in a top down fashion. That is, starting at an energy corre- 


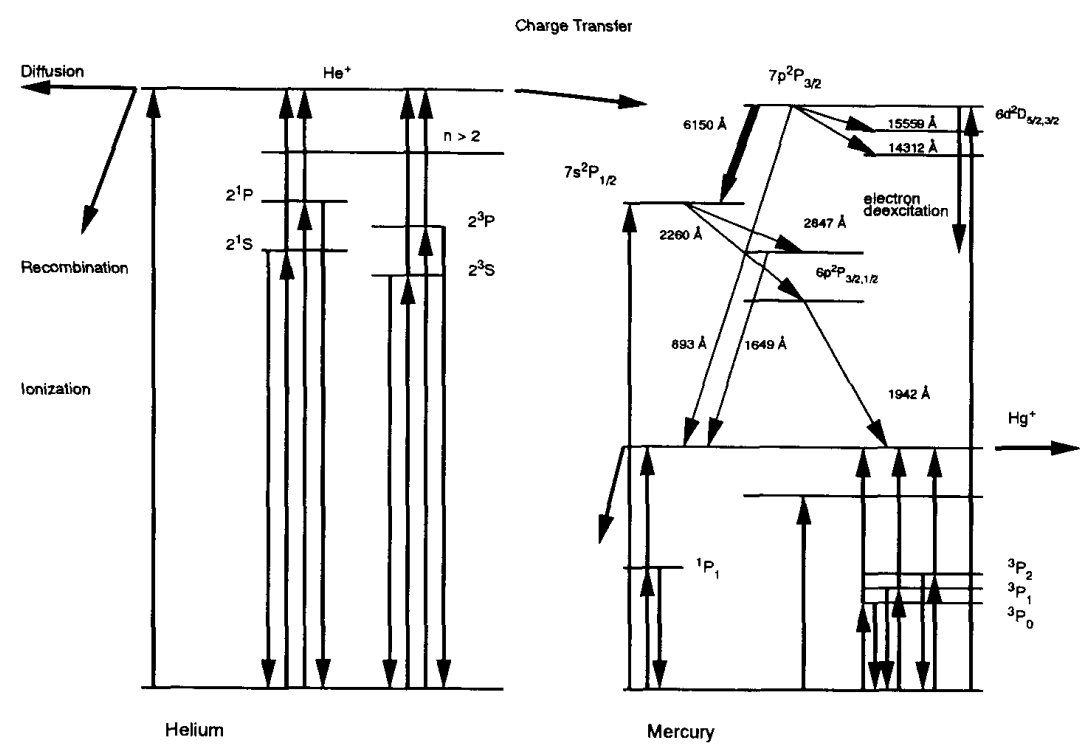

Fig. 3. Partial energy level diagram for helium and mercury used in modeling the helium mercury laser.

sponding to the discharge voltage, the energy degradation of primary electrons due to collisions is tracked. The energy of secondary electrons created by ionization are computed according to the differential cross section for ionization. Knowledge of the flow of electrons into and out of an energy bin allows the calculation of the density of electrons in that bin. The details of this calculation procedure are given in [20] and [26]. Both inelastic electronatom and elastic electron-atom, electron-ion, and electron-electron collisions have been included in the electron energy distribution calculations. In computing the electron energy distribution the width of the energy bins was $1 \mathrm{eV}$. No attempt was made to more highly resolve the energy distribution of the large group of electrons below $1 \mathrm{eV}$. Rather a mean electron temperature of $T_{e}=1800$ $\mathrm{K}$ was used to describe the thermalized group of low energy electrons. This temperature is in the range of that measured in other electron-beam sustained discharges [12], [24], [38] and it provides good agreement between the discharge characteristics of the model and experiment.

Fig. 3 is a partial energy level diagram representing the twelve states in helium and mercury considered in the model. The inelastic electron-helium collisions included were excitation of the $2^{1} S, 2^{3} S, 2^{1} P, 2^{3} \mathrm{P}$ states in helium and of a single pseudostate representing all helium levels of principle quantum number greater than 2 , as well as single and two step ionization reactions (i.e., ionization of metastable atoms). The inelastic electron-mercury collisions considered include excitation of the $6^{1} P_{1}$ and $6^{3} P_{0,1,2}$ states, and of a pseudostate representing higher energy levels, as well as ionization of the ground and excited state populations. Secondary electrons created by ionization were included in the accounting procedure. The effect of Penning type ionization reactions was also considered in calculating the electron energy distribution.
The electron energy distribution was used to calculate electron collisional excitation and ionization rates. A set of coupled rate equations was used to solve for the population densities of 12 states in helium and mercury. The densities of helium ions $N_{6}$ and mercury ions $N_{13}$ are coupled by the charge transfer reaction:

$$
\mathrm{He}^{+}+\mathrm{Hg} \rightarrow \mathrm{He}+\mathrm{Hg}^{+}+\Delta E
$$

and are calculated using,

$$
\begin{aligned}
\frac{d N_{6}}{d t}= & \sum_{i=0}^{4} R_{i, 6}+N_{1}^{2} p_{1,1} \\
& -N_{6}\left(s_{6}+N_{7} k_{6}+\beta N_{t}^{2}+\alpha N_{t}\right)=0 . \\
\frac{d N_{13}}{d t}= & N_{6} N_{7} k_{6}+\sum_{i=7}^{11} R_{i, 13}+N_{7} \sum_{i=1}^{2} N_{i} p_{i, 7} \\
& +N_{10}^{2} p_{10,10}-N_{13}\left(s_{13}+\beta N_{t}^{2}+\alpha N_{i}\right)=0 .
\end{aligned}
$$

Table I contains a collection of the notation used in the model. The total charge transfer cross section used in the model is that measured by Kano et al. [35] and is represented by $k_{6}$. Radiative and collisional recombination ion losses are represented by $\alpha$ and $\beta$, respectively. The results of Hennon et al. [36] and Collins et al. [37] were used for $\beta$ and $\alpha$, respectively. The Penning ionization coefficient $p_{1,1}$ is that given by Kolokeov and Pramaratov [39]. The third term in (3) represents the ionization of ground state mercury atoms by collisions with excited helium neutrals and the fourth term accounts for a Penning reaction between two $6^{3} P_{2}$ excited mercury neutrals. The cross sections used for the Penning reactions in (3) were those measured by Eliason and Hirshfelder [40]. 
TABLE I

Notation USED in MODEl. $N_{i}=$ density of $i$ th state where the states are defined as:

\begin{tabular}{llr}
\hline \multicolumn{1}{c}{ Specie } & \multicolumn{1}{c}{ State } & Index \\
\hline Helium & & \\
Ground State & $1 s^{21} S$ & 0 \\
Excited States & $1 s^{2} 2 s^{3} S$ & 1 \\
& $1 s^{2} 2 s^{1} S$ & 2 \\
& $1 s^{2} 2 s^{3} P$ & 3 \\
Lumped Excited State & $1 s^{2} 2 s^{1} P$ & 4 \\
Ion Ground State & $1 s n>2$ & 5 \\
Mercury & $1 s^{2} S$ & 6 \\
Ground State & & \\
Excited States & $6 s^{21} S$ & 7 \\
& $6 p^{3} P_{0}$ & 8 \\
& $6 p^{3} P_{1}$ & 10 \\
Lumped Excited State & $6 p^{3} P_{2}$ & 11 \\
Ion Ground State & $6 p^{1} P_{1}$ & 12 \\
Lower Laser Level & $6 s^{2} S_{1 / 2}$ & 13 \\
Upper Laser Level & $7 s^{2} S_{1 / 2}$ & 14 \\
\hline
\end{tabular}

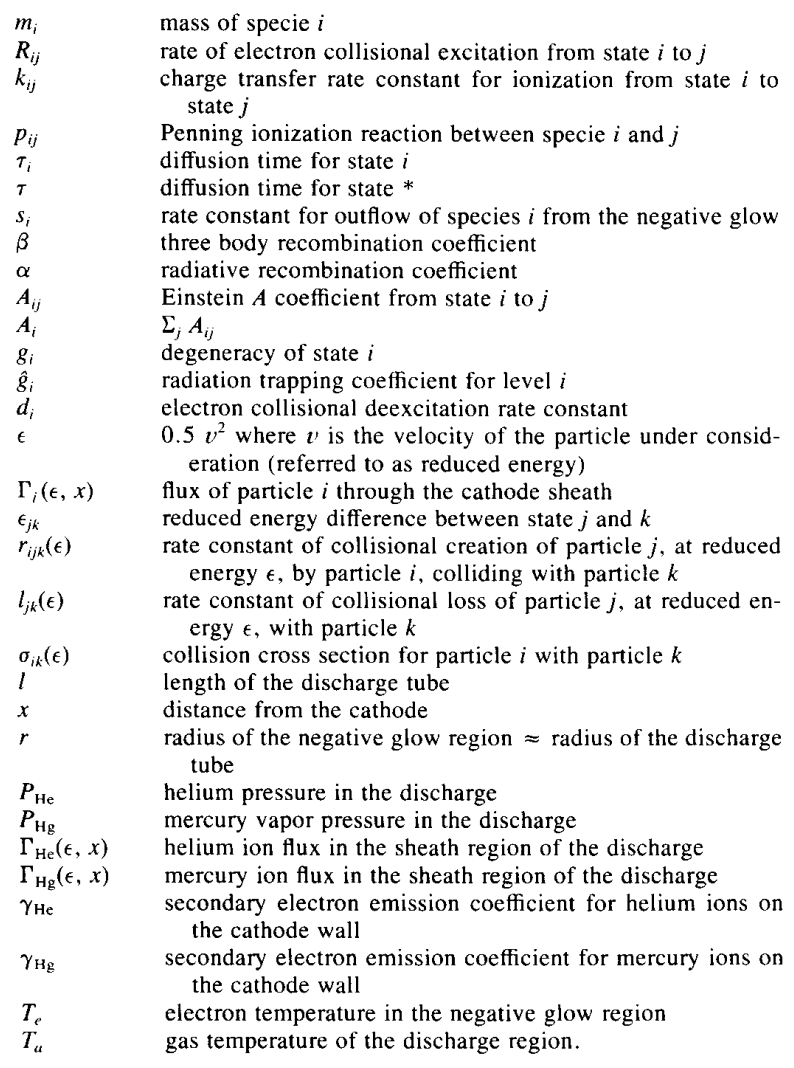

By assuming charge neutrality and neglecting doubly ionized species in the negative glow region the electron density is computed as

$$
N_{t}=N_{6}+N_{13} \text {. }
$$

In order to provide estimates of the effects of ionization of the low lying excited states of helium and mercury, a set of rate equations for these states was included in the model. In the case of the helium excited state system the $2^{1} S, 2^{3} S, 2^{1} P, 2^{3} P$ levels have been lumped together to form a single pseudostate whose density is denoted $\mathrm{He}^{*}$. The population of this state is calculated using,

$$
\begin{aligned}
\frac{d \mathrm{He}^{*}}{d t}= & -\left(\mathrm{He}^{*}\right)^{2} p_{11}-\mathrm{He}^{*}\left\{N_{7} p_{*^{7} 7}+d^{*}+\frac{1}{\tau^{*}}+r_{*}, 6\right\} \\
& +R_{\mathrm{ex}}+N_{6}\left(\beta N_{t}^{2}+\alpha N_{t}\right)=0
\end{aligned}
$$

Here $R_{\mathrm{ex}}, r_{*, 6}$ and $d^{*}$ represent electron collisional excitation, ionization, and deexcitation, respectively, and $\tau^{*}$ is the diffusion lifetime. Once the lumped excited state density is calculated, three fourths of the population was assumed to correspond to the triplet states, with half of the population corresponding to the $2^{3} S$ state. This assignment reflects experimental measurements that show that the $2^{3} S$ is clearly more heavily populated in a negative glow discharge [12]. The approximation is further justified by the results presented here which indicate that the helium excited states play an insignificant role in determining the laser characteristics of the device. The primary motivation for using this approximation was to reduce the computational complexity associated with solving equations for individual populations; a process which is complicated by collisional mixing between the levels.

The equations used for computing the mercury excited state densities are of the form,

$$
\frac{d N_{i}}{d t}=R_{i}-N_{i}\left(d_{i}+r_{e, i}+\hat{g}_{i} A_{i}+\frac{1}{\tau_{i}}+\sum_{j} N_{i} p_{i, j}\right)=0 \text {. }
$$

Here, $r_{e, i}$ represents the rate constant for electron collisional ionization of the excited state and $\hat{g}_{i}$ is the radiation trapping factor calculated using the method developed by Holstein [41].

Electron collisional deexcitation of excited states is primarily due to the coldest group of electrons in the negative glow. Due to the high frequency of elastic collisions, the low energy end of the distribution can be approximated by a Maxwellian energy distribution $f(E)$ with a mean temperature $T_{e}$. The collisional deexcitation rate constant is calculated by

$$
d_{i}=\left\{\frac{2}{m_{e}}\right\}^{1 / 2} \sum_{j} \int_{E} f(E) Q_{i j}^{*}(E) E^{1 / 2} d E
$$

where $Q_{i j}^{*}$ is the deexcitation cross section from level $i$ to $j$.

\section{B. Laser Level Population Densities}

The helium ion ground state is resonant in energy with the $7 p^{2} P_{3 / 2}$ state of $\mathrm{Hg}$ II which is the upper laser level of the $6150 \AA$ transition. Consequently, the charge transfer reaction (1) provides a selective mechanism for the excitation of the upper level of this transition which terminates on the $7 s^{2} S_{1 / 2}$ lower level. The resulting popu- 
lations of these states are used to calculate laser gain and optical output power. Fig. 3 also contains the mercury ion radiative processes included in calculation of the population densities of the laser levels.

The rate equations describing the populations of the laser upper and lower levels are given by:

$$
\frac{d N_{15}}{d t}=R_{7,15}+N_{6} N_{7} k_{6,15}-N_{15}\left(A_{15}+d_{15}\right)=0
$$

and

$$
\begin{aligned}
\frac{d N_{14}}{d t}= & R_{7,14}+N_{6} N_{7} k_{6,14}+N_{15}\left(A_{15,14}+d_{15,14}\right) \\
& -N_{14}\left(A_{14}+d_{14}\right)=0 .
\end{aligned}
$$

Here $k_{6,15}$ is the charge transfer rate constant from the helium ground state ion to the mercury ion laser upper level. Individual charge transfer branching ratios to the mercury ion excited states were calculated using the work of Johnsen et al. [42].

In the early stages of $\mathrm{He}-\mathrm{Hg}$ laser development researchers suggested that a Penning reaction between the $2^{3} S$ helium excited state and the $6 p^{3} P_{0}$ and $6 p^{3} P_{2}$ mercury excited states was a primary mechanism for excitation of the laser upper level [3]. However, in afterglow experiments Kano et al. [35] found no relation between the destruction rate of the helium excited state as a function of mercury density and the $6150 \AA$ spontaneous emission decay rate, thereby concluding that Penning reactions do not play an important role in the excitation of the laser upper level. As a result this reaction has been excluded from the laser level equations.

The spontaneous emission coefficient for the laser lower level has been measured by Semenova and Smirnov [43]. Experimentally measured radiative lifetimes of the laser upper level are not available in the literature. Consequently, estimates for this and other lifetimes in the mercury ion were obtained from published results of BatesDamgaard calculations [44] as well as from an unpublished work based on a model developed by researchers at Oxford University [45].

Consideration was also given to the possibility that radiation from the laser lower level might be trapped. To compute a trapping factor for the $7 s$ radiative lifetime required the $6 p^{2} P_{3 / 2,1 / 2} \mathrm{HgII}$ populations to be included in the calculation. However, for all the discharge conditions considered here population of the $6 p^{2} P_{3 / 2,1 / 2}$ levels is sufficiently low such that trapping of the radiation from the $7 s$ level is not significant.

\section{The Negative Glow Cathode Sheath Interface}

The drift of ions across the negative glow cathode sheath boundary was determined by equating the outflow of ions from the negative glow (e.g., for helium $\left.\pi r^{2} l N_{6} S_{6}\right)$ to the flux of ions entering the sheath $\left(N_{6} v_{6} 2 \pi r l\right)$. Assuming that the ions entering the sheath arrive at the boundary with a perpendicular velocity equal to the Bohm velocity $v_{6}$ and that losses from the ends of the plasma cylinder can be neglected, the outflow rate constant for helium ions is given by [46]

$$
s_{6}=\frac{2 v_{6}}{r} .
$$

Consequently the fluxes of helium and mercury ions at the sheath glow boundary are given by

$$
\begin{gathered}
\Gamma_{6}\left(\epsilon_{b}, x_{b}\right)=N_{6} v_{6} \\
\Gamma_{13}\left(\epsilon_{b}, x_{b}\right)=N_{13} v_{13}
\end{gathered}
$$

where $\epsilon_{b}$ is the ion reduced energy corresponding to the Bohm velocity and $x_{b}$ represents the boundary between the sheath and the negative glow. The ion energy corresponding to the Bohm velocity is small compared to the energy imparted to the ions by the strong field in the sheath and consequently is of little significance in calculations regarding the sheath region.

On the other hand, electrons accelerated through the sheath arrive at the negative glow boundary with substantial energies. The flux of electrons into the negative glow is converted to a source term in the Boltzmann equation for electrons. For a discussion of the formalism used to solve the Boltzmann equation see [26]. Using the notation of [26] the source term is calculated using

$$
S(E)=\frac{2 \Gamma_{e}\left(\frac{E}{m_{e}}, x_{b}\right)}{r} .
$$

\section{The Cathode Sheath Region}

The energy spectrum of fast neutral atoms and ions, and the electric field distribution in the sheath, are calculated by solving equations of continuity and by Poisson's equation respectively. A one-dimensional model structure similar to that developed by McClure [28] is used to model the cathode sheath region. In this formalism the sheath is divided into a discrete set of $N$ cells, the boundaries of which make up equipotential surfaces. Based on the charge density in a cell, the electric field strength and the cell width were calculated using Poisson's equation in conjunction with the assumption that the entire discharge voltage is dropped over the sheath. Because of the interdependence of the cell width and the electric field strength, the solution procedure is iterative.

Within each equipotential cell the energy distributions of the ion and electron fluxes are calculated. Charged particles gain energy from the electric field as they move from one cell to the next while energy is lost through various types of collisions. Table II summarizes the collisional processes included in the model of the sheath region.

The charge density in the sheath is calculated from the continuity equations for ions. Because electron velocities are much higher than that of ions, electron space charge does not contribute much to the electric field and consequently is neglected. The space charge distribution is cal- 
TABLE II

Collision Processes Included in the Sheath Model

\begin{tabular}{ll}
\hline \multicolumn{1}{c}{ Reaction } & \multicolumn{1}{c}{ Process } \\
\hline $\mathrm{He}^{+}+\mathrm{He} \rightarrow \mathrm{He}+\mathrm{He}^{+}$ & Ion-neutral charge transfer \\
$\mathrm{He}^{+}+\mathrm{Hg} \rightarrow \mathrm{Hg}^{+}+\mathrm{He}$ & Ion-neutral charge transfer \\
$\mathrm{He}^{+}+\mathrm{He} \rightarrow \mathrm{He}^{+}+\mathrm{He}$ & Momentum transfer \\
$\mathrm{He}^{+}+\mathrm{He} \rightarrow \mathrm{He}^{+}+\mathrm{He}^{+}+e$ & Ionization by fast ions \\
$\mathrm{He}+\mathrm{He} \rightarrow \mathrm{He}^{+}+\mathrm{He}^{+}+e$ & Ionization by fast neutrals \\
$\mathrm{Hg}++\mathrm{Hg} \rightarrow \mathrm{Hg}+\mathrm{Hg}^{+}$ & Ion-neutral charge transfer \\
$e+\mathrm{He} \rightarrow \mathrm{He}^{*}+e(\Delta \epsilon)$ & Electron impact excitation \\
$e+\mathrm{Hg} \rightarrow \mathrm{Hg}^{*}+e(\Delta \epsilon)$ & \\
$e+\mathrm{He} \rightarrow \mathrm{He}^{+}+2 e$ & Electron impact ionization \\
$e+\mathrm{Hg} \rightarrow \mathrm{Hg}^{+}+2 e$ & Electron impact ionization \\
\hline
\end{tabular}

culated by

$$
\rho(x)=q \int_{0}^{\epsilon_{m}}\left\{\frac{1}{v_{6}(\epsilon)} \frac{\delta \Gamma_{6}(\epsilon, x)}{\delta \epsilon}+\frac{1}{v_{13}(\epsilon)} \frac{\delta \Gamma_{13}(\epsilon, x)}{\delta \epsilon}\right\} d \epsilon
$$

and the resulting electric field is given by Poisson's equation,

$$
\frac{d E(x)}{d x}=-\frac{\rho(x)}{\epsilon_{o}}
$$

The variation of the flux of particle type $j$ traveling through the sheath is calculated using the continuity equation, [28]

$\frac{\delta \Gamma_{j}(\epsilon, x)}{\delta x}=-\frac{q E}{m_{j}} \frac{\delta \Gamma_{j}(\epsilon, x)}{\delta \epsilon}+\sum_{i} \sum_{k} N_{k} r_{i j k}(\epsilon)-N_{j} \sum_{k} l_{j k}(\epsilon)$

where the creation of particle $j$, at reduced energy $\epsilon$, is calculated as

$$
r_{i j k}(\epsilon)=\Gamma_{i}\left(\epsilon+\epsilon_{j k}, x\right) \sigma_{i k}\left(\epsilon+\epsilon_{j k}\right) .
$$

Here $\epsilon_{j k}$ is the reduced threshold energy for an inelastic collision. Likewise the destruction of particles $j$ at a reduced energy of $\epsilon$ is given as

$$
l_{j k}(\epsilon)=\Gamma_{j}(\epsilon, x) \sigma_{j k}(\epsilon) .
$$

Ions and secondary electrons created by ionization are assumed to be released at zero energy. This is justifiable because the great majority of secondary electrons are created with energies that are small compared with the energy imparted by the electric field in the cathode sheath. The production of these particles is represented as a flux with the minimum kinetic energy accounted in the model $\epsilon_{\min }$. Thus

$\Gamma_{j}\left(\epsilon_{\min }, x\right)=\frac{1}{q E} \int_{0}^{\epsilon_{m}} \sum_{i} \sum_{k} m_{k} N_{k}\left(r_{i j k}(\epsilon)+r_{e j k}(\epsilon)\right) d \epsilon$

where $r_{e j k}$ represents the creation of specie type $j$, by electron impact with particle $k, \epsilon_{m}$ is the maximum reduced energy, which is defined by the position and the electric field in the cathode sheath. The flux of electrons through the sheath is denoted as $\Gamma_{e}(\epsilon, x)$. Electron emission from the cathode is assumed to result only from secondary emission caused by the impinging ion fluxes and is calculated using,

$$
\Gamma_{e}\left(\epsilon_{a t}, 0\right)=\int_{0}^{q V_{f}} \gamma_{6} \frac{\delta \Gamma_{6}(\epsilon, 0)}{\delta \epsilon}+\gamma_{13} \frac{\delta \Gamma_{13}(\epsilon, 0)}{\delta \epsilon} d \epsilon .
$$

Here $\epsilon_{a v}$, represents the reduced energy at which electrons are emitted from the cathode and $\gamma_{6}$ and $\gamma_{13}$ represent the secondary electron emission coefficients for helium and mercury ion bombardment. $V_{f}$ is the discharge voltage. Actually secondary electron emission also results from photons and neutral species impinging on the surface. However, for the discharge conditions considered here photoemission is much smaller than emission due to excited neutrals and ions [47] and consequently is neglected. Emission of electrons due to bombardment of the cathode by fast ground state neutral fluxes is also not significant at the low impact energies characteristic of this type of discharge [22]. Secondary emission coefficients for excited species and ions are comparable. However experimental measurements of these types of discharges have shown that excited state densities are typically 1 to 3 orders of magnitude smaller than the ion densities [12], justifying the approximation of neglecting the contribution due to excited species.

Secondary emission coefficients of low energy helium ions $\gamma_{6}$ for a variety of cathode materials have been reported in the literature and ranges from 0.2 to 0.4 [23], [48], [49]. The emission coefficient for mercury ions on various materials has been measured at high energies by Ismail [50] and Schwartz and Copeland [51]. Unfortunately, the secondary emission coefficients of helium and mercury on stainless steel have not been measured at the low ion energies considered here.

Zalm and Becker [52] offer an approximate formula for the secondary emission coefficient based on the ionization potential of the impinging ion, and the work function and Fermi energy of the cathode material:

$$
\gamma_{i}=\frac{0.2\left(0.8 E_{i}-2 \phi\right)}{\epsilon_{F}}
$$

where $\epsilon_{F}, \phi$, and $E_{i}$ are the Fermi energy, work function of the cathode material, and the ionization energy of the impinging ion, respectively. Karashaev et al. [53] have measured the work function of steel to be $4.85 \mathrm{eV}$. However, work by Surplice and D'Arcy [54] indicates that in the presence of an electric field, such as the strong field in the cathode sheath, the effective work function of steel may be reduced by as much as $1.6 \mathrm{eV}$.

Taking the ratio of the secondary emission coefficients, due to helium and mercury ion bombardment, to eliminate the Fermi energy, using the reduced work function, and choosing a value of 0.3 for the helium coefficient (20) yields a value of 0.044 for the mercury coefficient. These 
values were used to obtain the results presented in this paper. The sensitivity of the model to ratio of the emission coefficients was also studied and is summarized in a later section.

In the model, secondary electrons emitted at the cathode surface, are tracked in a fashion similar to ion flow towards the cathode. Inelastic collisions with helium and mercury atoms are responsible for electron energy loss while traveling through the sheath. The same inelastic collision mechanisms used in the calculation of the electron energy distribution in the negative glow region are considered in the sheath. Here, the excited states of atomic helium and mercury are lumped together to form a single pseudostate for each species. Since the density of excited atoms in the sheath region is not of interest in these calculations, but rather the effect of inelastic energy transfer on the electron energy distribution, this approximation is reasonable and simplifies computational aspects significantly.

The accuracy of this cathode sheath modeling scheme has been verified by experimental measurements. The electric field distribution in the cathode sheath region of a high voltage electron-beam sustained discharge was calculated and compared to measurements obtained by Stark laser spectroscopy. In most cases the agreement between the calculated and measured electric field values was better than $10 \%[55]$.

\section{E. Gas Temperature}

To estimate the densities of the neutral atomic species in the discharge volume it is necessary to include the effects of collisional gas heating. To accomplish this the heat equation, [56]

$$
\nabla \cdot \lambda_{a} \nabla T_{g}=H
$$

was solved, for the gas temperature using finite difference techniques. The thermal conductivity of the gas is denoted as $\lambda_{a}$ and $H$ represents gas heating terms due to elastic collisions of neutral gas atoms with electrons in the negative glow and with ions in the cathode sheath. For the results presented here the cathode was assumed to be maintained at $700 \mathrm{~K}$. Calculated temperature profiles are relatively flat across the radius of the discharge tube with a $50 \%$ temperature difference between the center of the discharge and the cathode being typical. To simplify the computational burden of a spatially varying gas density we have assumed a uniform average temperature in the discharge based on the value obtained from the solution of (21).

\section{F. Optical Gain and Laser Power}

At the discharge conditions of interest both Doppler and collisional broadening make significant contributions to the gain profile of the lasing transitions. Consequently, it was necessary to account for the gain saturation process by using a convolution of Gaussian and Lorentzian line shape functions. The saturated gain for the laser transition is given as, [57]

$$
\begin{aligned}
\gamma\left(\nu, I_{\nu}\right)= & \frac{A_{15,14} \lambda^{2}}{8 \pi}\left(N_{15}-\frac{g_{15}}{g_{14}} N_{14}\right) \frac{\Delta \nu_{h}}{\Delta \nu_{H}} \int_{0}^{\infty} p(f) \\
& \cdot \frac{\Delta \nu_{H}}{2 \pi\left((\nu-f)^{2}+\left(\Delta \nu_{H} / 2\right)^{2}\right)} d f
\end{aligned}
$$

where

$$
p(f)=\left\{\frac{4 \ln 2}{\pi}\right\}^{1 / 2} \frac{1}{\Delta \nu_{D}} \exp \left\{-4 \ln 2\left(\frac{f-\nu_{0}}{\Delta \nu_{D}}\right)^{2}\right\} .
$$

The convolved lineshape has a width given by

$$
\Delta \nu_{H}^{2}=\Delta \nu_{h}^{2}\left(1+\frac{I_{v}}{I_{s}}\right)
$$

and $\Delta \nu_{h}$ is the Lorentzian full width half maximum linewidth. The saturation intensity $I_{s}$ can be expressed as

$$
\begin{aligned}
I_{s} & =E_{0} / g\left(\nu_{0}\right) \\
E_{0} & =\frac{8 \pi h \nu_{0} A_{15} A_{14}}{\left(A_{14}+A_{15}-A_{15,14}\right) A_{15,14} \lambda^{2}}
\end{aligned}
$$

and $g\left(\nu_{0}\right)$ is a Lorentzian line shape function evaluated at the laser line center.

The laser output intensity is related to the intracavity intensity by the transmission $T_{o}$ of the output coupler.

$$
P_{\text {out }}=I_{\nu} T_{o} \pi \omega_{m}^{2}
$$

where $\omega_{m}$ is the assumed Gaussian beam radius. The laser output power is calculated using a numerical bisection routine which converges to the laser cavity intensity which makes the round-trip optical gain equal to cavity losses. The cavity losses were estimated by taking into account both mirror transmission and diffraction. $\mathrm{TEM}_{j j}$ modes corresponding to values of $j \leq 7$ were found to have sufficient gain to oscillate for the conditions considered here. The optical power contained in these modes was included in the calculation of laser output power. An output coupler with $1 \%$ transmissivity and a totally reflective rear mirror, both of $1 \mathrm{~m}$ curvature, were selected to allow a comparison between the model results and the experimental results of Piper and Webb [6].

\section{G. Electron Collision Cross Sections}

Table III contains a list of references compiled to provide the necessary electron collision cross sections used in the model.

\section{Results}

In the preceding section, the hollow cathode discharge helium-mercury laser model was divided into four physical/computational sections. Hereafter, results obtained through modeling of the hollow cathode discharge laser are presented in the same fashion. First, the discharge current-voltage characteristics are discussed. Character- 
TABLE III

REFERENCES FOR ELECTRON COLLISIONAL CROSS SECTION INFORMATION

\begin{tabular}{lc}
\hline Ionization & \\
Helium & {$[58]-[63]$} \\
Mercury & {$[64]-[68]$} \\
Excitation & \\
Helium & {$[69]-[75]$} \\
Mercury & {$[76]$} \\
Momentum Transfer & {$[77]-[79]$} \\
Laser Level Excitation & {$[80]$} \\
\hline
\end{tabular}

istics of the cathode sheath region are then examined followed by a discussion of the negative glow region. Finally, the dependence of laser power of the $6150 \AA \mathrm{Hg}$ II transition on the discharge current, buffer gas pressure, and metal vapor density are discussed.

\section{A. The Self-Sustained Discharge}

In laboratory hollow cathode discharges it is observed that a threshold voltage exists below which the discharge is not self-sustained. That is, as the voltage applied across the device is lowered the number of ions produced by each electron that is emitted from the cathode decreases until the discharge quits. When the number of electrons emitted from the cathode cannot create a sufficient density of ions to in turn maintain the same secondary electron cathode emission the discharge current will decay and eventually cease. The threshold voltage value is dependent on total gas pressure and partial pressures of the fill gases, and the cathode material and geometry. Above this threshold the discharge current increases rapidly with increasing voltage.

These characteristics of a self-sustained discharge are well described by the model. Fig. 4 is a plot of the current-voltage characteristics of the HCD as predicted by the model. If the selected voltage is too low to sustain a discharge, the current calculated by the model steadily decreases until the computer job times out. The model threshold voltage values are similar to those observed in laboratory devices. For the cathode material considered here, stainless steel, the secondary electron emission coefficient due to mercury is much lower than that of helium. Consequently as the ratio of the mercury density to the helium density is increased a higher threshold voltage is observed. However, the ratio of the two gas densities does not entirely determine the effect. As can be seen in Fig. 4 , increasing the mixing ratio by lowering the buffer gas pressure has much less effect on the voltage threshold value than increasing the metal vapor density.

To determine the sensitivity of the model to the electron emission coefficient of the cathode runs were also made using a $\mathrm{Hg}$ emission coefficient of 0.088 . The curve with the open diamond data symbol in Fig. 4 is the current-voltage curve computed using an assumed $\gamma_{13}$ of 0.088 . As can be seen here, increasing the emission coefficient by a factor of two reduces the discharge voltage necessary to obtain a certain current by approximately

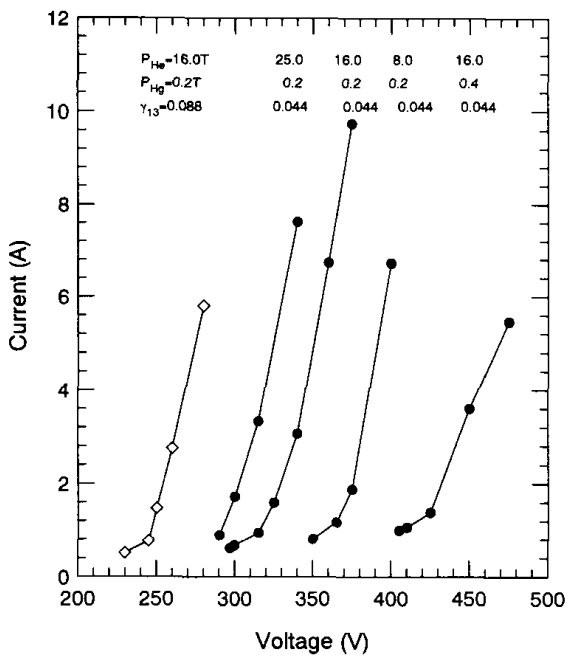

Fig. 4. Current-voltage characteristics for the hollow cathode discharge as predicted by the model. The helium pressure and mercury vapor pressure as well as the secondary electron emission coefficient used to produce the results are listed above the respective curve.

$25 \%$, yielding a discharge voltage threshold of less than $230 \mathrm{~V}$. Piper and Webb noted that the threshold voltage necessary to sustain a discharge for their device was approximately $300 \mathrm{~V}$ in pure helium and rose to a value in excess of $320 \mathrm{~V}$ upon introduction of the metal vapor [81]. The value of the secondary electron emission coefficient of mercury on stainless steel of 0.044 , estimated as discussed in Section II-D yields results which are consistent with experiment.

\section{B. The Cathode Sheath}

1) Ion Fluxes: Fig. 5(a) illustrates the net ion flux to the cathode as a function of the buffer gas pressure for a constant discharge power density of $150 \mathrm{~W} \cdot \mathrm{cm}^{-3}$. Note that a fourfold increase of the buffer gas pressure results in slightly less than a factor of two increase in the helium ion and mercury ion fluxes at the cathode. On the other hand in Fig. 5(b) it can be seen that an increase in the mercury vapor density results in a decrease in the helium ion flux to the cathode and a slight decrease in the mercury ion flux. These characteristics are a result of the charge transfer reaction between helium ions and mercury ground state atoms. Increasing the mixing ratio by increasing the metal vapor density has the effect of providing more ground state mercury atoms for charge transfer reactions, effectively reducing the helium ion flux to the cathode. Decreasing the mixing ratio by increasing the buffer gas pressure has a smaller effect because a large portion of any additional helium ions produced as a result rapidly undergo a charge transfer reaction.

Fig. 6 shows the evolution of energy spectra of the ion fluxes as they propagate through the sheath from the negative glow towards the cathode. The sheath-glow interface is at the forward portion of the figure and the cathode is located at the origin. The spike at the high energy end 


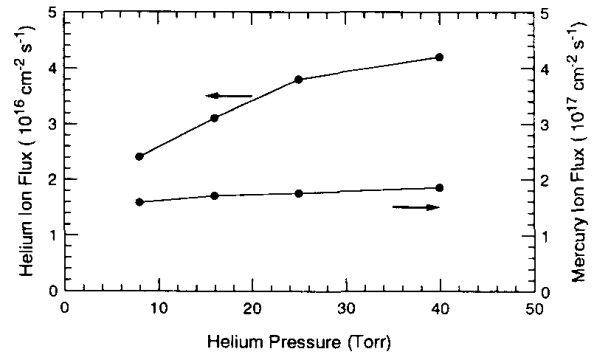

(a)

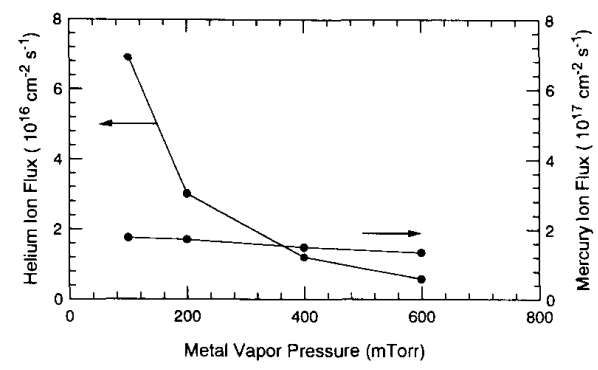

(b)

Fig. 5. Net ion fluxes to the cathode. (a) $P_{\mathrm{Hg}}=0.2$ torr. (b) $P_{\mathrm{He}}=16$ torr.

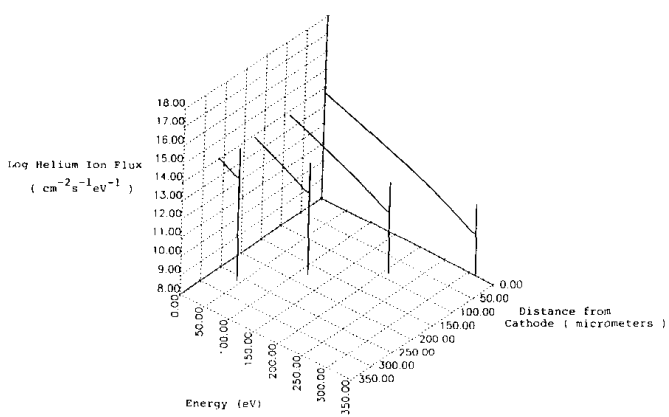

(a)

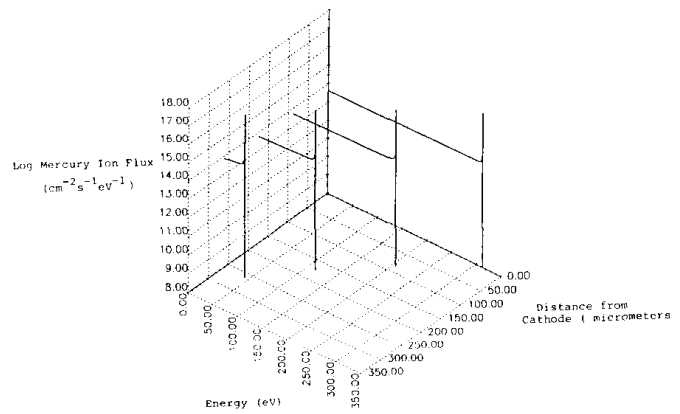

(b)

Fig. 6. Spatial evolution of the ion fluxes during propagation through the cathode sheath. (a) Helium ion flux. (b) Mercury ion flux. $P_{\mathrm{He}}=16$ torr, $P_{\mathrm{Hg}}=0.2$ torr. $V_{f}=314 \mathrm{~V}$.

of the flux spectrum is made up of ions which have not collided during their flight through the cathode sheath. Typically, this beam component of the ion fluxes makes up a few percent of the total ion flux to the cathode. The beam component of helium ion flux decreases as it propagates through the sheath while the mercury flux does not. This is again a consequence of the charge transfer reaction between helium ions and ground state mercury.

2) Electric Field Distribution: The electric field in the cathode sheath region of a glow discharge has been the subject of much research in the last five years [28], [29], [55]. Fig. 7(a) is a plot of the calculated sheath electric field of the HCD for three buffer gas pressures at a constant voltage and metal vapor density. At low pressures the field can be characterized by a nearly linear decrease across most of the sheath with a sharp nonlinear drop at the glow-sheath boundary. This result is in good agreement with the cathode sheath electric field measurements made using laser Stark spectroscopy by Lee et al. [55] and those made with electron beam probing by Warren [82]. In addition the shape of our electric field profile agrees with the computed profiles of Bayle et al. [30].

At high buffer gas pressures the field is not as linear in the sheath region. The total device current increases significantly when the buffer gas pressure is increased between 8 and 25 torr at the voltage used to generate the results in Fig. 7(a). Consequently the space charge in the sheath for the high pressure discharge condition is much larger than that of the low pressure discharge causing the nonlinear portion of the field to extend over the majority of the cathode fall region. The increasing nonlinearity of the electric field as the pressure is raised has been observed experimentally in lower pressure, high voltage (13 torr) glow discharges, where the cathode sheath dimension is on the order of $1 \mathrm{~cm} \mathrm{[55].} \mathrm{Measurement} \mathrm{of} \mathrm{the}$ electric field in the cathode fall for the higher pressure conditions studied here is complicated by the rather small sheath dimensions. For the range of conditions used to generate Fig. 7(b), the cathode sheath thicknesses are only 100 to $400 \mu \mathrm{m}$, making optical probing difficult.

An increase in buffer gas pressure decreases the cathode sheath width. As the gas pressure is increased at a fixed voltage, the discharge current increases significantly resulting in a higher space charge density in the cathode sheath and consequently in a narrower sheath. A similar effect is also evident in the results shown in Fig. 7(b) which is a plot of the cathode sheath width as a function of the discharge voltage for three buffer gas pressures. As the discharge voltage is increased, at a fixed buffer gas pressure, the ion current through the sheath increases resulting in a larger space charge concentration and a smaller cathode sheath.

3) Electron Flux: Fig. 8 illustrates the energy distribution of the electron flux as it propagates through the cathode sheath. The comb like structure in the high energy region of the electron flux is due to the existence of beam electrons which have undergone inelastic collisions with either a helium or a mercury atom. The various spikes in the flux spectrum occur at the energy difference between the beam energy and the threshold energies for excitation and ionization of the atoms present. This distinct 


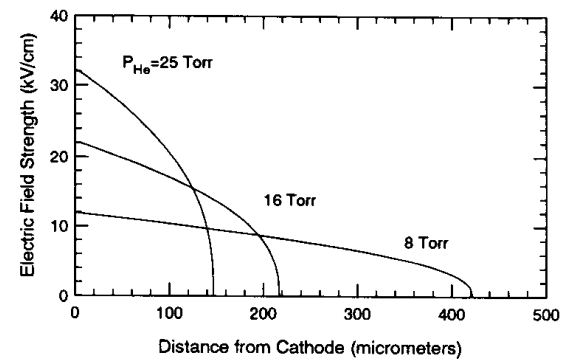

(a)

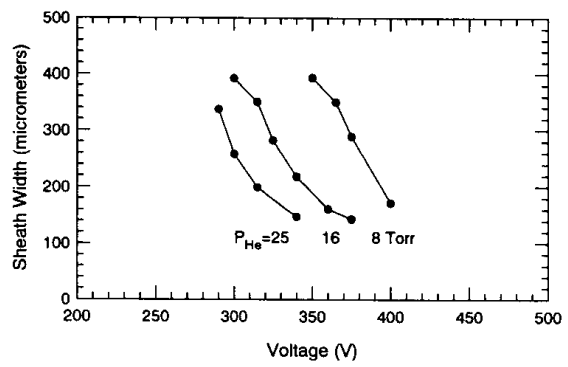

(b)

Fig. 7. Cathode sheath electric field strength and cathode sheath width for three buffer gas pressures. (a) Electric field strength for a discharge voltage of $340 \mathrm{~V}$ and a mercury vapor pressure of 0.2 torr. (b) Sheath width as a function of discharge voltage. Here the mercury vapor pressure was 0.2 torr.

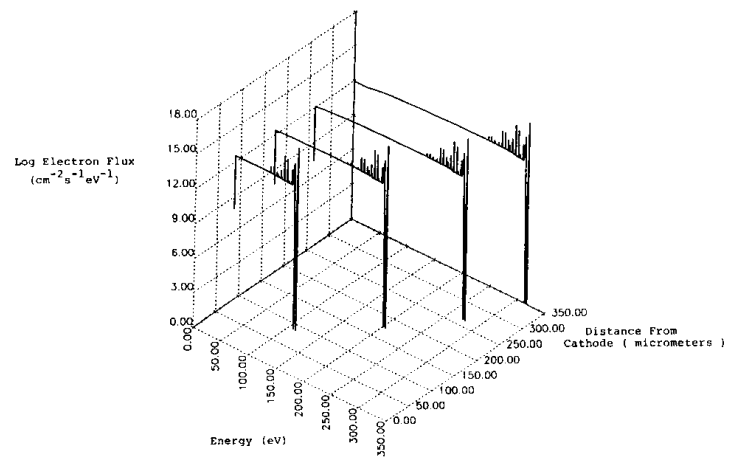

Fig. 8. Evolution of the electron flux energy spectrum during propagation through the cathode sheath. $V_{f}=314 \mathrm{~V}, I=0.93 \mathrm{~A}, P_{\mathrm{He}}=16$ torr, $P_{\mathrm{Hg}}$ $=0.2$ torr.

structure results from assuming that electrons emitted from the cathode have a monoenergetic distribution with a reduced energy of $\epsilon_{a}$. If instead a distribution with a spread in energy of a few $\mathrm{eV}$, such as that measured by Hagstrum [48] were used the distinct features in the flux spectrum would be smoother as seen in the results computed by Carman for the cathode sheath in an argon glow discharge [83]. Moreover if the energy of secondary electrons created by ionization in the sheath was not assumed to be zero but rather with a distribution defined by the differential cross section the distinct features in the high energy region of the spectrum would be further smoothed.

A parameter of interest in the study of hollow cathode discharges is the electron-beam generation efficiency. The
TABLE IV

Electron-Beam Generation EfFiciency. THE Discharge Voltage WaS $340 \mathrm{~V}$ and the Mercury Vapor Pressure WAS 0.2 TORR

\begin{tabular}{ccr}
\hline Current (A) & Helium Pressure (torr) & $\eta_{e}(\%)$ \\
\hline 0.67 & 8.0 & 8.25 \\
3.06 & 16.0 & 9.45 \\
7.62 & 25.0 & 11.01 \\
\hline
\end{tabular}

flux of energetic electrons into the negative glow region plays an important role in the level of ionization of the discharge. Also, in charge transfer laser schemes the ionization rate of the buffer gas determines, to a large extent, the excitation rate of the laser upper level. The electronbeam generation efficiency can be defined as:

$$
\eta_{e}=\frac{A m_{e} \int_{0}^{q V_{f}} \epsilon \cdot \frac{\delta \Gamma_{e}\left(\epsilon, x_{b}\right)}{\delta \epsilon} d \epsilon}{V_{f} I}
$$

where $A$ is the cathode area, $I$ is the discharge current, $V_{f}$ the discharge voltage, and $x_{b}$ is the sheath-glow boundary. Table IV contains the computed electron beam generation efficiency for three discharge conditions. The efficiencies are all in the vicinity of $10 \%$. This relatively low efficiency is primarily a result of the low secondary emission coefficient of the cathode material and of the energy with which the ions bombard the cathode. At these ion energies electron emission from the cathode is primarily due to potential emission. The beam generation efficiency is much higher in multikilovolt glow discharges [84]. Operation at higher voltages results in higher energy ions bombarding the cathode causing kinetic emission of electrons. The use of high secondary electron yield cathode materials in multikilovolt glow discharges has been shown to produce dc electron beams with efficiencies up to $80 \%$ [84]. As a result, some charge transfer metal vapor lasers excited by high voltage electron-beam glow discharges are almost an order of magnitude more efficient than the hollow cathode laser operating on the same transition [17].

\section{The Negative Glow Region}

The energy distribution of the input electron flux into the negative glow and the collisional term in the Boltzmann equation determine the electron energy distribution in this region of the discharge. Fig. 9(a) is a plot of the calculated electron energy distribution averaged over the volume of the negative glow region of the HCD. The distribution has features which resemble those of the electron flux energy spectrum in the sheath. The large beam component of the discharge, apparent at the high energy end of the distribution, is due to an input flux of electrons at the full cathode fall energy.

Immediately below the beam component is a relatively low density region which is primarily comprised of elec- 


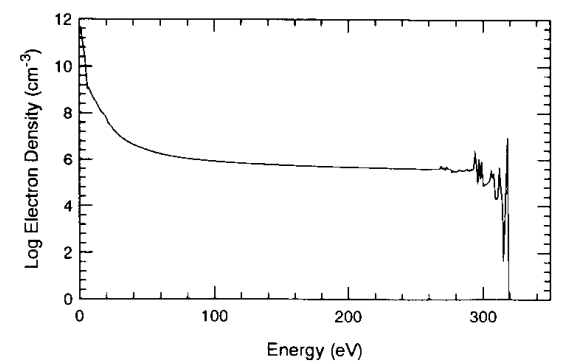

(a)

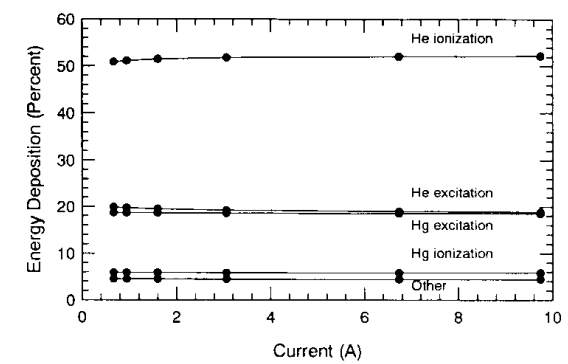

(b)

Fig. 9. Electron energy distribution and electron-beam energy deposition in the negative glow region for fill pressures of $P_{\mathrm{Hc}}=16$ torr and $P_{\mathrm{Hg}}=$ 0.2 torr. (a) Here the discharge conditions were $V_{f}=314 \mathrm{~V}, I=0.93 \mathrm{~A}$ (b) Proportion of electron-beam energy deposited in various collision mechanisms.

trons which traveled through the sheath without colliding, but have undergone ionizing collisions with excited state atoms in the negative glow region. Below this low density region there is some additional structure in the distribution, corresponding to electrons having undergone an inelastic collision with a ground state buffer gas or metal vapor atom resulting in an excited atom or an ion. In each of these events the electron loses a discrete amount of energy. The various peaks in the distribution in this region occur at an energy equal to the difference between the electron beam energy and the excitation and ionization threshold energies for helium and mercury. The production of secondary electrons during ionizing collisions smooths out the central part of the electron energy distribution and is the primary cause for the large electron density component at low energies [26].

Fig. 9(b) is a plot of the relative fraction of the electron-beam energy deposited in the various collision processes in the negative glow. As seen here, the hollow cathode discharge is attractive for exciting charge transfer metal vapor laser transitions because of the relatively large portion of electron energy deposited in ionization of the buffer gas even in the presence of metal vapor concentrations. For the discharge conditions considered here approximately $50 \%$ of the electron-beam energy is deposited in ionization of the buffer gas. A significant portion of this energy is subsequently deposited in the laser upper levels of metal ions by charge transfer reactions. The majority of the remainder of the electron beam energy goes into collisional excitation of ground state helium and mercury atoms, with a rather small amount, $7 \%$, deposited in collisional ionization of mercury atoms.

Another interesting feature is the uniformity of the energy deposition associated with each collision mechanism over a wide range of currents. As previously mentioned, once the discharge has been established a relatively small increase in voltage results in a large change in current. As a result the variation of voltage required to generate the range of currents in Fig. 9(b) is fairly small. The electron collisional cross sections for the various energy deposition channels change little over the energies corresponding to this voltage variation, and as a result the relative deposition percentages do not change appreciably.

1) Ion Densities: In the HCD modeled in this work the mercury ion density is typically an order of magnitude higher than the helium ion density because the charge transfer reaction rapidly depletes the ground state helium ion density in the negative glow region. Fig. 10 is a plot of the ion densities as a function of discharge current. For all the discharge conditions considered in this work single step electron collisional ionization of the ground state is the dominant helium ionization mechanism. Electron impact ionization of the helium metastable states accounts for only a fraction of a percent of the total ionization rate. Penning ionization by collisions of two $2^{3} S$ helium atoms is also negligible for the conditions considered here. The creation of mercury ions is dominated by the helium mercury charge transfer reaction which simultaneously excites several states in $\mathrm{Hg}$ II. Approximately $14.5 \%$ of the total charge transfer rate goes into excitation of the upper level of the $6150 \AA$ transition in singly ionized mercury [36], [42]. The remainder of the charge transfer rate populates other excited states such as the $7 p^{2} P_{1 / 2}$ state which constitutes the upper level of the $7945 \AA$ laser transition of $\mathrm{Hg}$ II.

\section{Laser Output Characteristics}

Fig. 11 is a plot of the calculated laser output power of the $6150 \AA$ mercury ion transition as a function of the discharge current. The experimental measurements of Piper and Webb are included for comparison. The laser power calculated by the model and the experimental results are in good agreement, except at high currents where model results show power saturation. Electron deexcitation of the laser upper level is responsible for the saturation exhibited in the model results. In experiments carried out by Piper and Webb [6] the hollow cathode was operated in a direct current fashion up to currents of only 3.5 A while the model results were obtained assuming $\mathrm{CW}$ operation at all currents. As a result the discharge conditions between the model and the experiment are expected to be somewhat different above $3.5 \mathrm{~A}$.

Also shown in Fig. 11 is the calculated laser efficiency. Here the efficiency has been defined as the ratio of laser output power on the $6149.5 \AA$ transition to the product of the discharge current and voltage. The turnover in ef- 


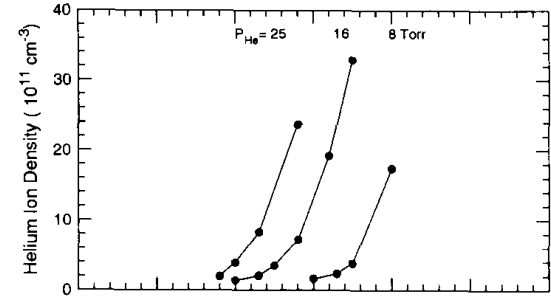

(a)

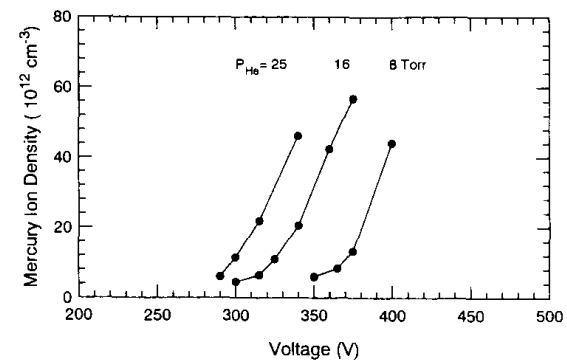

(b)

Fig. 10. Helium and mercury ion densities in the negative glow regions of the hollow cathode discharge for different buffer gas pressures.

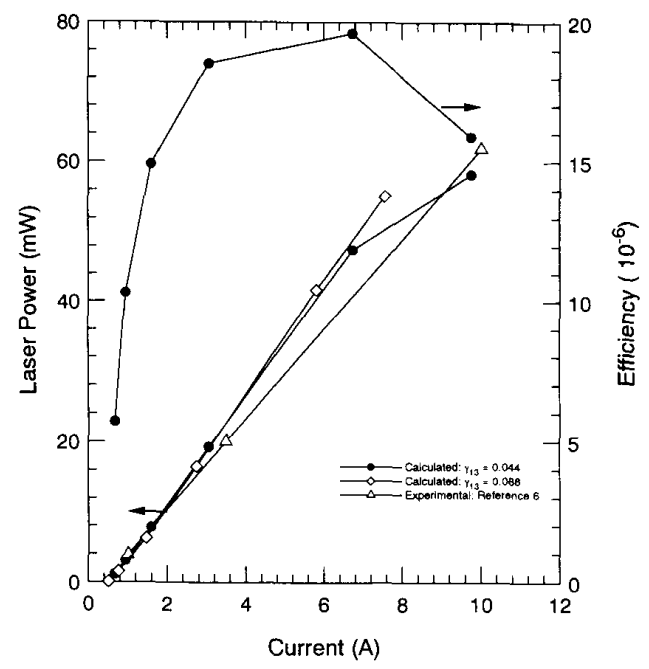

Fig. 11. $6150 \AA$ laser output power and laser efficiency as a function of discharge current, $P_{\mathrm{He}}=16$ torr, $P_{\mathrm{Hg}}=0.2$ torr.

ficiency at high currents is due to the saturation of the laser output power. These results were obtained assuming the resonator configuration described previously.

The data plotted with open diamond symbols in Fig. 11 represent the laser power predicted by the model using a secondary electron emission coefficient of 0.088 . In terms of laser power, raising the secondary electron emission coefficient has little effect. However, this does not mean that the secondary emission coefficient is not an important parameter in determining the laser characteristics. The effect of an increase in the secondary emission coefficient is to lower the voltage at which a given value of the laser output is obtained, thereby resulting in an increased laser efficiency.

Fig. 12(a) is a plot of the laser output power dependence on helium gas pressure at a mercury pressure of 0.2 torr and a discharge current of $1 \mathrm{~A}$. The experimental results of Piper and Webb in the figure were obtained at a reservoir temperature of $115^{\circ} \mathrm{C}$, corresponding to a mercury vapor pressure of approximately 0.4 torr. Both the experimental and model results show that the laser power peaks at a buffer gas pressure near 16 torr. The rise of the laser output power to the maximum is due to increased excitation of the laser upper level through the charge transfer reaction between the helium ion ground state and neutral mercury atoms. As the pressure is increased, the proportion of discharge energy being deposited in ionization of helium increases and results in greater charge transfer excitation of the laser upper level. Raising the buffer gas pressure beyond the maximum results in significant pressure broadening of the gain profile, resulting in a drop in the laser output power.

The model described herein assumes a spatially uniform negative glow, and consequently does not take into account the reduced reaching distance of the beam electrons with increasing pressure. This effect can cause a depletion of laser upper level excitation in the center of the discharge and consequently a drop in output power. However, a simple calculation done assuming an unperturbed electron trajectory after a collision, shows that the beam electrons should have a reaching distance of approximately 5 times the radius of the discharge tube at a buffer gas pressure of 40 torr. Nonetheless, at discharge pressures above 20 torr nonuniform ionization due to the reaching distance effect may explain the somewhat lower laser powers observed in the experiment.

The dependence of laser output power on the metal vapor density is illustrated in Fig. 12(b). To allow a comparison, the experimental results of Piper and Webb have been included by using the vapor pressure for mercury calculated from the mercury side arm temperatures given in [6]. However, in making this comparison it should be considered that in the experiments described in [6] a slow helium flow through the discharge was maintained to assure an even distribution of metal vapor in the active region, consequently it is likely that the mercury vapor pressure in the discharge was not completely determined by the sidearm temperature. The vapor pressure given by the sidearm temperature could be higher than that in the actual discharge.

At a discharge current of $1 \mathrm{~A}$ and a helium pressure of 16 torr the model predicts an optimum mercury partial pressure of 0.2 torr. The rise of the laser power with increasing mercury density is a result of an increased population inversion as more metal atoms become available. The peak power is obtained at a point where the charge transfer from helium ions is a maximum. The decrease in laser power beyond this point is a result of the decreased proportion of discharge energy being deposited in the ion- 


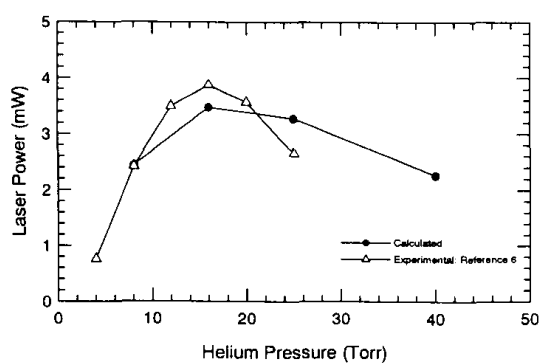

(a)

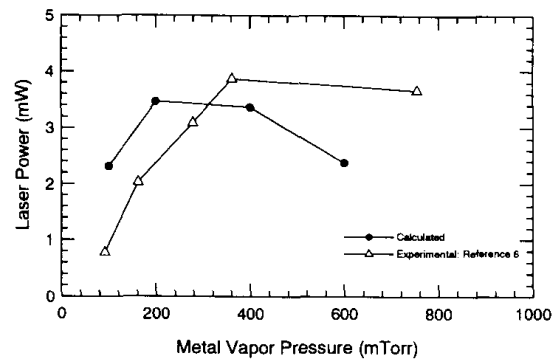

(b)

Fig. 12. $6150 \AA$ laser power dependence on buffer gas pressure and metal vapor density. (a) Here the discharge conditions were $I=1 \mathrm{~A}, P_{\mathrm{Hg}}=0.2$ torr. (b) Here the discharge conditions were $I=1 \mathrm{~A}, P_{\mathrm{He}}=16$ torr.

ization of helium. As the metal vapor density is increased, the proportion of energy deposited in collisional excitation and ionization of mercury increases. Since charge transfer is the primary upper laser level excitation mechanism a reduction in the energy deposited into ionization of helium results in a reduced excitation rate of the upper laser level and consequently a reduced laser output power.

\section{Summary}

A model of a negative glow discharge laser has been developed based on a self-consistent treatment of the cathode sheath and the negative glow regions of the discharge. The electron energy distribution and population densities in the negative glow are calculated self-consistently with the electric field distribution and the energy spectra of the charged particle fluxes in the cathode. The model has been applied to the helium-mercury HCD laser operating on the $6149.5 \AA$ transition of $\mathrm{Hg}$ II.

The model simulates well the general dependence of the discharge current on applied voltage and the minimum voltage required to maintain a self-sustained discharge. As expected, the characteristics of the helium-mercury HCD are shown to be heavily influenced by the charge transfer reaction which depletes the helium ion concentration and increases the mercury ion density. Consequently, the flux of mercury ions to the cathode is typically an order of magnitude higher than that of the helium ions. However, the much larger secondary electron emission coefficient of helium ions compared to that of mercury ions results in both ion species playing an important role in cathode electron emission. The efficiency of generation of beam electrons in the HCD is calculated to be in the vicinity of $10 \%$. This relatively low value is one of the primary factors limiting the laser efficiency. The relative concentration of mercury and helium not only influences electron emission but also determines the channels through which the electron energy is deposited in the discharge. At optimum conditions for laser oscillation slightly more than $50 \%$ of the electron energy is deposited in the ionization of helium and approximately $14 \%$ of it is selectively transferred to the $7 p^{2} P_{3 / 2}$ laser upper level. The calculated variation of the laser output power as a function of discharge current, helium pressure and mercury vapor density is in good agreement with experimental measurements reported in the literature.

The treatment of the cathode sheath discussed here, by including the consideration of kinetic electron emission which is dominant in glow discharges having kilovolt sheath voltage drops, can also accurately describe the generation of dc electron beams in glow discharges. Consequently, the model described herein can be modified to study other types of cw negative glow lasers, such as those pumped by multikilovolt electron beams. In these lasers the glow discharge electron guns are placed on the optical axis at the ends of a plasma tube which contains the negative glow. The plasma tube is held at anode potential and the negative glow is immersed in an axial magnetic field. Extending the model discussed here to describe high voltage negative glow $\mathrm{cw}$ electron beam lasers requires relatively minor modification if the plasma can be assumed to be axially uniform. This is a good assumption in the case in which the plasma tube is short in comparison with the reaching distance of two counter-propagating electron beams. The interface between the cathode sheath and the negative glow regions needs to be modified to take into account the different geometry of the device. In addition, the influence of the magnetic field on charged particle diffusion needs to be included in modeling the negative glow.

\section{ACKNOWLEDGMENT}

The authors acknowledge the assistance of Dr. M. Christon and J. Cooley for their expert assistance in developing code used in the simulations and the Colorado State University Computing Center for providing large blocks of supercomputer time.

\section{REFERENCES}

[1] C. S. Willet, Introduction to gas discharge lasers: Population inversion mechanisms. New York: Pergamon, 1974

[2] R. L. Byer, W. E. Bell, E. Hodges, and A. L. Bloom, "Laser emission in ionized mercury: Isotope shift, linewidth, and precise wavelength," J. Opt. Soc. Amer., vol. 55, p. 1598, 1965.

[3] W. K. Schuebel, "Continuous visible and near infrared laser action in HgII," IEEE J. Quantum Electron., vol. QE-7, 1971.

[4] R. C. Jensen, G. J. Collins, and W. R. Bennett Jr., "Low noise cw hollow-cathode zinc ion laser," Appl. Phys. Lett., vol. 18, p. 50, 1971.

[5] M. Janossy Csillag, V. Rosa, and T. Salamon, "New infrared cw laser oscillation in Cu II,'” Phys. Lett. A, vol. 50, p. 13, 1974.

[6] J. A. Piper and C. E. Webb, "Power limitations of the cw He-Hg laser," Opt. Commun., vol. 13, p. 122, 1975. 
[7] J. R. McNeil, G. J. Collins, H. B. Persson, and D. L. Franzen, "CW laser oscillation in Cu II," Appl. Phys. Lett., vol. 27, p. 595, 1975. [8] R. D. Reid, J. R. McNeil, and G. J. Collins, "New ion laser transitions in He-Au mixtures," Appl. Phys, Lett, vol. 29, p. 666, 1976.

[9] K. Rosza, M. Janossy, L. Csillag, and J. Bergou, " $\mathrm{Cw}$ cu II laser in a hollow anode-cathode discharge," Opt. Commun., vol. 28, pp. $162-$ $164,1977$.

[10] W. L. Johnson, J. R. McNeil, G. J. Collins, and C. B. Persson, "CW laser action in the blue-green spectral region from Ag II," Appl. Phys. Lett., vol. 29, p. 172, 1976. For a discussion of unidentified silver ion laser transitions see R. D. Reid, D. C. Gerstenberger, J. R. McNeil, and G. J. Collins, Appl. Phys. Lett., vol. 48, p. 3994, 1977.

[11] H. J. Eichler, H. J. Koch, J. Salk, and G. Schafer, "Performance of CuII lasers with cylindrical hollow cathodes," IEEE J. Quantum Electron., vol. QE-15, p. 9, 1979.

[12] J. R. McNeil, "New sputtered metal vapor laser systems," Ph.D. dissertation, Colorado State University, 1979.

[13] D. C. Gerstenberger, R. Solanki, and G. J. Collins, "Hollow cathode metal ion lasers," IEEE J. Quantum Electron., vol. QE-16, p. 820, 1980.

[14] H. J. Eichler, H. Koch, R. Molt, and J. L. Qiu, “Optimization of the uv Cull laser," Appl. Phys. B., vol. 26, pp. 49-56, 1981.

[15] R. Solanki, "Optimization of power and mode structure in a metal ion hollow cathode laser," Ph.D. dissertation, Colorado State University, 1982.

[16] J. Mizeraczyk, M. Neiger, and J. Steffer, "Comparison of He-Cd ${ }^{+}$ white-light laser oscillations in longitudinal and transverse hollowcathode tubes," IEEE J. Quantum Electron., vol. QE-20, pp. 12331235,1984

[17] J. J. Rocca, J. D. Meyer, and G. J. Collins, "1 W zinc ion laser," Appl. Phys. Lett., vol. 43, pp. 37-40, 1983.

[18] J. J. Rocca, " $\mathrm{CW}$ recombination laser in a flowing negative glow plasma," Appl. Phys. Lett., vol. 47, p. 1145, 1985, and J. J. Rocca, H. L. Mancini, and B. Wernsman, "Cd recombination laser in a plasma generated by an electron beam," IEEE J. Quantum Electron., vol. QE-22, p. 509, 1986.

[19] B. Wernsman, J. J. Rocca, and H. L. Mancini, "CW ultraviolet and visible laser action from ionized silver in electron beam generated plasma," IEEE Photon. Technol. Lett, vol. 2, pp. 12-15, 1990.

[20] B. E. Warner, "Investigation of the hollow cathode discharge at high current density," Ph.D. dissertation, University of Colorado, 1979.

[21] Y. M. Kagan, R. I Lyagushchenko, and S. N. Khvorostovskii, "Electron energy distribution in a hollow cathode," Sov. Phys. Tech. Phys., vol. 17, p. 1346, 1973. See also Sov. Phys. Tech. Phys., vol. 18, p. 943, 1974; Sov. Phys. Tech. Phys., vol. 20, p. 1164, 1976; Sov. Phys. Tech. Phys., vol. 20, p. 1167, 1976.

[22] H. D. Hagstrum, "Theory of auger ejection of electrons from metals by ions," Phys. Rev., vol. 96, p. 195, 1954.

[23] M. Kaminsky, Atomic and ionic impact phenomena on metal surfaces. New York: Academic, 1965.

[24] K. B. Persson, "Brush Cathode Plasma-A well behaved plasma," J. Appl. Phys., vol. 36, p. 3086, 1965.

[25] P. R. Caron and F. Russo, "Some measurements of primary electrons in the helium negative glow," J. Appl. Phys., vol. 41, p. 3547, 1970.

[26] G. J. Fetzer, J. J. Rocca, G. J. Collins, and R. Jacobs, "Model of cw argon ion lasers excited by low energy electron beams," J. Appl. Phys, vol. 60, p. 2739, 1986.

[27] W. D. Davis and T. A. Vanderslice, "Ion energies at the cathode of a glow discharge," Phys. Rev., vol. 131, p. $219,1963$.

[28] G. W. McClure and K. D. Granzow, "High-voltage discharges in D gas. II. cathode fall theory," Phys. Rev., vol. 125, p. 3, 1962.

[29] E. A. Den Hartog, D. A. Doughty, and J. E. Lawler, "Laser optogalvanic and fluorescence studies of the cathode region of a glow discharge," Phys. Rev. A., vol. 38, p. 2471, 1988.

[30] P. Bayle, J. Vacquie, and M. Bayle, "Cathode region of a transitory discharge in $\mathrm{CO}_{2}$; I. Theory of the cathode region," Phys. Rev. A., vol. 34 , p. 360,1986 .

[31] B. E. Warner, K. B. Persson, and G. J. Collins, "Metal vapor production by sputtering in a hollow-cathode discharge: Theory and experiment," J. Appl. Phys., vol. 50, p. 5694, 1979.

[32] W. E. Bell, " "Visible laser transitions in $\mathrm{Hg}^{+}, "$ Appl. Phys. Lett. vol. 4 , p. $34,1964$.

[33] A. Ferrario, Opt. Commun., vol. 7, p. 376, 1973.

[34] L. R. Peterson, "Discrete deposition of energy by electrons in gases," Phys. Rev., vol. 187, p. 105, 1967.

[35] T. Shay Kano and G. J. Collins, "A second look at the excitation mechanism of the He- $\mathrm{Hg}^{+}$laser," Appl. Phys. Lett., vol. 27, p. 610, 1975 .

[36] E. Hennon and J. Hirscherg, "Electron ion recombination in dense plasmas," Phys. Rev., vol. 125, p. 795, 1962.

[37] C. B. Collins, A. S. Hicks, W. E. Wells, and R. Burton, "Measurement of the rate coefficients for the recombination of He with electrons," Phys. Rev. A., vol. 6, p. 1545, 1972.

[38] Z. Yu, J. J. Rocca, G. J. Collins, and C. Y. She, "The energy of thermal electrons in electron beam created helium discharges," Phys. Lett., vol. 96A, p. 125, 1983.

[39] N. B. Kolokeov and P. M. Pramaratov, "Interaction between metastable atoms in a helium afterglow," Sov. Phys. Tech. Phys., vol 23 , p. 176,1978 .

[40] M. Eliason and J. Hirshfelder, "General collision theory for the rate of bimolecular gas phase reactions," J. Chem. Phys., vol. 30, p. $1426,1959$.

[41] T. Holstein, "Imprisonment of resonance radiation in gases," Phys. Rev., vol. 83, p. 1159,1951

[42] R. Johnsen, M. T. Leu, and M. A. Biondi, "Studies of nonresonant charge transfer between atomic atoms and ions," Phys. Rev. A, vol 8, p. 1808,1973 .

[43] I. V. Semenova and Y. M. Smirnov, "Determination of excitation cross sections and transition probabilities of $\mathrm{HgIl}$," Opt. Spectrosc. vol. 44, p. $245,1978$.

[44] I. M. Littlewood, J. A. Piper, and C. E. Webb, "Excitation mech anisms in cw He-Hg lasers," Opt. Commun., vol. 16, p. 45, 1976.

[45] B. P. Das and G. J. Fetzer, Unpublished work, 1986.

[46] F. F. Chen, Introduction to plasma physics and controlled fusion: Volume I Plasma Physics. New York: Plenum, 1984.

[47] M. Garbuny, Optical Physics. New York: Academic, 1965.

[48] H. D. Hagstrum, "Auger ejection of electrons from molybdenum by noble gas ions," Phys. Rev., vol. 104, p. 672, 1956.

[49] Y. Takeishi and H. D. Hagstrum, "Auger-type electron ejection from the (111) face of nickel by slow $\mathrm{He}^{+}, \mathrm{Ne}^{+}$, and $\mathrm{Ar}^{+}$ions," Phys. Rev., vol. 137, p. A641, 1965.

[50] H. Ismail, "Etude du taux de pulverisation et du coefficient d'emission electronique secondaire de cibles metalliques polycristallines bombardees par des ions $\mathrm{Hg}^{+}$, dans un domaine e'energie allant de 5A, $30 \mathrm{keV}, "$ Revue de Physique Appliquee, vol. 5, p. 759, 1970.

[51] M. Schwartz and P. L. Copeland, "Secondary emission by positive ion bombardment," Phys. Rev., vol. 96, p. 1466, 1954.

[52] P. C. Zalm and L. J. Beckers, "Ion induced secondary electron emission from copper and zinc,"' Suf. Sci., vol. 152/153, p. 135, 1985.

[53] A. A. Karashaev, V. K. Kumykov, L. G. Belgorokov, A. M. Kokov, and Kh. M. Guketlov, "Surface tension and electron work function of steel Kh18N10T in the solid state," Fiz. Mezhfaznykh Yavlenii, vol. 2, p. 49,1977

[54] N. A. Surplice and R. J. D'Arcy, "Reduction in the work function of stainless steel by electric fields," J. Phys. F, vol. 2, p. L8, 1972.

[55] S. A Lee, L. U A Anderson, J. J. Rocca, M. Marconi, and N. D. Reesor, "Electric field distribution in the cathode sheath of an electron beam glow discharge,"' Appl. Phys. Lett., vol. 51, p. 409, 1987.

[56] M. J. Kushner and B. E. Warner, "Large-bore copper vapor lasers: Kinetics and scaling issues, "J. Appl. Phys., vol. 54, pp. 2970-2982, 1983.

[57] J. T. Verdeyen, Laser Electronics. Englewood Cliffs, NJ: PrenticeHall, 1981.

[58] J. Bretagne, G. Delouya, J. Godart, and V. Puech, "High energy electron distribution in an electron-beam-generated plasma," J. Appl. Phys., vol. 14, p. 1225, 1981.

[59] D. Rapp and P. Englander-Golden, "Total cross sections for ionization and attachment in gases by electron impact: I. Positive ionization," J. Chem. Phys., vol. 43, p. 1464, 1965.

[60] L. R. Peterson and J. E. Allen, Jr., "Electron impact cross sections for argon," J. Chem. Phys., vol. 56, p. 6068, 1972.

[61] M. Dixon, H. Harrison, and A. Smith, "A measurement of the electron impact ionization cross section of helium metastable states, " $J$. Phys. B., vol. 9, p. $2617,1976$.

[62] R Sovie and J. Dugan, NASA Technical Note D-3/2I, Nov, 1965.

[63] L. Vriens and R. Keijser, "Ionization processes in the positive column of the low pressure Hg-Ar discharge," J. Appl. Phys., vol. 49, p. 3807,1978

[64] J. W. Liska, "Efficiencies of ionization of helium and mercury by electron impact at high voltages,"' Phys. Rev., vol. 46, p. 169, 1934.

[65] P. Smith, "The ionization of mercury vapor by electron impact," Phys. Rev., vol. 37, p. 808, 1931. 
[66] R. Abrines, I. C. Percival, and N. A. Valentine, "Classical cross sections for ionization of hydrogen atoms by electrons,'" Proc. Phys. Soc. London, vol. 89, p. 515, 1966.

[67] H. Harrison, Thesis, Catholic University of America Press Inc., Washington DC, 1956.

[68] J. Jobe and R. St. John, "Absolute measurements of the $2^{i} P$ and $2^{3} P$ electron excitation cross sections of helium atoms," Phys. Rev., vol. 164, p. $117,1967$.

[69] W. Fon, K. Berrington, P. Burke, and A. Kingston, "The $1{ }^{1} S \rightarrow 2{ }^{3} S$ and $1^{1} S \rightarrow 2^{3} P$ excitation of helium by electron impact," $J$. Phys. $B$, vol. 12, p. $1861,1979$.

[70] S. Trajmar, "Differential and integral cross sections for the excitation of the $2^{3} S, 2^{3} S$, and $2^{3} P$ states of He by electron impact at 29.6 and $40.1 \mathrm{eV}, "$ Phys. Rev. A, vol. 8, p. 191, 1973.

[71] J. K. Rice et al., "Effect of charge polarization on inelastic scattering: Differential and integral cross sections for excitation $2^{1} S$ state of helium by electron impact," Phys. Rev. A, vol. 5, p. 762, 1972.

[72] R. Hall, J. Reinhardt, G. Jorez, and J. Mazeau, "Near threshold electron impact excitation functions for the $n=2$ states of helium by the trapped electron method," J. Phys. B, vol. 5, p. 66, 1972.

[73] F. M. Pichanick and J. A. Simpson, "Resonances in the total cross sections for metastable excitation of noble gases by electron impact,' Phys. Rev., vol. 168, p. 64, 1968.

[74] F. Donaldson, M. Hender, and J. McConkey, "Vacuum ultraviolet measurements of the electron impact excitation of helium," $J$. Phys. B., vol. 5, p. $1192,1972$.

[75] G. B. Crooks, R. DuBois, D. Golden, and M. Rudd, "Observation of a broad resonance in the $2^{3} S$ excitation of helium by electron impact," Phys. Rev. Lett., vol. 29, p. 327, 1972.

[76] L. J. Keiffer, "A compilation of electron collision cross section data for modeling gas discharge lasers," JILA Information Center Report, No. 13, Boulder, CO, Sept. 30, 1973.

[77] L. Frost and A. V. Phelps, "Momentum-transfer cross sections for slow electrons in $\mathrm{He}, \mathrm{Ar}, \mathrm{Kr}$, and $\mathrm{Xe}$ from transport coefficients," Phys. Rev., vol. 136, p. 1538, 1964.

[78] B. McClure, "Diffusion experiments to determine certain cross sections for collisions between electrons and gases,"' Nucl. Sci. Abstr., vol. 18, p. $13,1964$.

[79] P. Ganas, S. Dutta, and A. E. S. Green, "Modified Born approximation and the elastic scattering of electrons from helium," Phys. Rev. $A$, vol. 2, p. 111, 1970.

[80] 1. Semenova and Y. Smirnov, "Determination of excitation cross sections and transition probabilities of Hg II," Opt. Spectrosc., vol. 44, p. $245,1978$.
[81] J. A. Piper and C. E. Webb, "A hollow cathode device for $\mathrm{cw}$ helium-metal vapour laser systems,"'J. Phys. D., vol. 6, p. 400, 1973

[82] R. Warren, Phys. Rev., vol. 98, p. 1650, 1955.

[83] R. J. Carman, "A simulation of electron motion in the cathode sheath region of a glow discharge in argon," J. Phys. D, vol. 22, p. 55 , 1989.

[84] J. J. Rocca, J. D. Meyer, Z. Yu, M. Farrell, and G. J. Collins, "Multikilowat electron beams for pumping $\mathrm{cw}$ ion lasers," Appl. Phys. Lett., vol. 41, p. 811, 1982; J. J. Rocca, J. D. Meyer, M. R. Farrell, and G. J. Collins, "Glow discharge electron beams: cathode materials, electron guns design and applications," J. Appl. Phys., vol. 56, p. 790, 1984.

Gregory J. Fetzer (S'87-M'87) received the B.S., M.S., and Ph.D. degrees in electrical engineering from Colorado State University, Fort Collins, in 1980, 1982, and 1990 , respectively.

He is currently the New Product Scientist for Lear Siegler Measurement Controls Corporation, Littleton, CO. His broad range of research interests include laser applications to atmospheric measurements, remote sensing, point monitoring, spectroscopy, nonlinear optics, and gas discharges.

Dr. Fetzer is a member of the Optical Society of America.

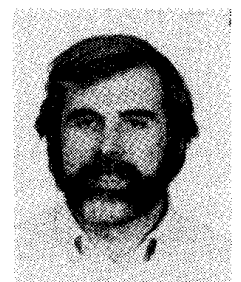

Jorge J. Rocca (S'80-M'83) received the diploma in physics from the University of Rosario, Argentina, in 1978, and the Ph.D. degree from Colorado State University, Fort Collins, in 1983.

$\mathrm{He}$ is currently an Associate Professor with the Department of Electrical Engineering at Colorado State University. His broad research interests include the development of new short-wavelength lasers and the physics of discharges and plasmas. His work has included the development of ion la sers excited by electron beams, the generation of intense electron beams from glow discharges, the demonstration of recombination lasing in negative glow plasmas, and the study of dense capillary discharges which he has proposed as possible source of short-wavelength recombination laser radiation.

Dr. Rocca received a National Science Foundation Presidential Young Investigator Award for 1985-1990 and is also a recipient of the Halliburton Foundation Award. He is a member of the Optical Society of America. 